\title{
Perfil de actividad científica de las universidades españolas en cuatro áreas temáticas: un enfoque multifactorial
}

\author{
María Bordons*, Rosa Sancho**, Fernanda Morillo*, Isabel Gómez*
}

Resumen: Este estudio analiza la actividad de las universidades españolas mediante datos de input y output científico, factores estructurales y datos del entorno regional, con el fin de explorar las relaciones entre variables. Se incide en las diferencias de comportamiento de las universidades en función de sus áreas de actividad, centrándose el análisis en las áreas de Química, Ingeniería, Ciencias Sociales y Ciencias Humanas. Se evidencian diferencias en los hábitos de publicación y colaboración según las áreas, y se analiza la influencia sobre la actividad universitaria de distintas factores como son el tamaño y la antigüedad de la universidad, su grado de especialización temática o el desarrollo industrial de su entorno.

Palabras clave: Universidad, especialización temática, indicadores bibliométricos, actividad científica.

\section{Scientific activity profile of Spanish universities in four thematic areas: a multifactor analysis}

Abstract: The activity of Spanish universities was analysed in terms of input data, scientific output, structural factors and regional development data, with the aim of exploring relationships between these variables. Special interest was paid to the different behaviour of universities with regard to their areas of activity, focusing on Chemistry, Engineering, Social Sciences and Human Sciences. Differences were detected in publication and collaboration patterns depending on the area of activity. The influence of factors such as the size and age of universities on their performance was examined, as was the effect of their degree of specialisation and the level of industrial development of their environment.

Keywords: University, subject specialisation, bibliometric indicators, research performance.

* Instituto de Estudios Documentales en Ciencia y Tecnología (IEDCYT), Centro de Ciencias Humanas y Sociales (CCHS), Consejo Superior de Investigaciones Científicas (CSIC), Madrid (España). Correo-e: maria.bordons@cchs.csic.es, fernanda.morillo@cchs.csic.es, isabel.gomez@cchs.csic.es

** Departamento de Publicaciones. Consejo Superior de Investigaciones Científicas (CSIC), Madrid (España).rsancho@cindoc.csic.es

Recibido: 20-3-09; 2. ${ }^{\text {a }}$ versión: 1-9-09; aceptado: 2-10-09. 


\section{Introducción}

El importante papel de la Universidad en el sistema educativo e investigador de los países y la necesidad de identificar aquéllas que sobresalen atendiendo a criterios de excelencia científica y docente, ha desencadenado en los últimos años la aparición de diversos estudios que comparan la actividad de las universidades a nivel internacional. En esta línea se sitúan los "rankings de universidades" surgidos recientemente (SJTU, 2005; THES, 2007), el primero elaborado en Shanghai y el segundo en el Reino Unido, que clasifican a las universidades atendiendo a criterios de prestigio, junto a datos de input y output, entre los cuales se incluyen algunos indicadores bibliométricos. Estas clasificaciones han despertado gran interés entre los gestores de investigación y el público en general, pero presentan algunas limitaciones que reducen su utilidad. Entre estas últimas se pueden mencionar diversas limitaciones metodológicas, como es el uso de indicadores unidimensionales, la dificultad de obtener índices compuestos válidos y fiables, la falta de rigor (Van Raan, 2005) o la consideración de las universidades independientemente de su orientación temática (Moed, 2006), lo que, sin duda, puede influir en su posición final en las clasificaciones.

La importancia de este último aspecto nos ha llevado a plantear el presente trabajo, cuyo objetivo es analizar la actividad científica de las universidades españolas, teniendo en cuenta los condicionantes estructurales de su entorno y en función de su especialización temática. El fin último de esta línea de trabajo es proporcionar información relevante a los gestores de la investigación a través de un mejor conocimiento de la influencia de distintos factores estructurales, funcionales y contextuales sobre la actividad universitaria.

Este trabajo es continuación de un estudio previo (Gómez y otros, 2009), centrado en el análisis de la actividad del sector universitario en España a través de indicadores clásicos de inversiones y resultados de la investigación, así como indicadores de docencia y estructurales, con el fin de analizar la interrelación entre estas variables. Dado que las disciplinas científicas difieren en las características de su investigación y en sus hábitos de publicación, y que el perfil temático de las universidades puede variar en función de las titulaciones ofertadas, se considera oportuno analizar separadamente cada área temática. El estudio actual desciende, pues, a análisis de áreas temáticas concretas, e introduce indicadores basados en publicaciones en revistas nacionales para obtener una visión más completa de la actividad investigadora en su doble vertiente nacional e internacional.

\section{El sistema universitario español}

En el curso 2008-09 el sistema universitario español contaba con 50 universidades públicas (incluyendo la UNED y la UIMP), y 24 universidades privadas (http://www.crue.org/), presentando algunas universidades públicas una larga 
historia desde su fundación (p. ej. la universidad de Salamanca fundada en 1218 y la de Valladolid en 1346).

La promulgación de la Ley de Reforma Universitaria (LRU) en 1983 constituye un hecho decisivo en la historia de la universidad española. Entre los cambios que introduce en la legislación se puede destacar el relativo a la autonomía universitaria, según la cual las universidades pasan a ser entidades autónomas con capacidad para establecer sus propios programas. Se transfiere la gestión administrativa y financiera de las universidades desde el Estado central a las 17 Comunidades Autónomas, que pasan a ser responsables últimas de las universidades públicas ubicadas en su región, terminando así el modelo de Estado centralista que había prevalecido en España durante décadas. Solamente la Universidad de Educación a Distancia, y la Universidad Internacional Menéndez y Pelayo, mantienen su dependencia del gobierno central.

Sin embargo, la I+D no está transferida a las diferentes Comunidades Autónomas, por lo que las áreas prioritarias de investigación se deciden de acuerdo con la política científica promovida por el Estado. Por otra parte, la financiación de la I+D para el sector de Educación Superior proviene, principalmente, de los Presupuestos Generales del Estado y, en forma creciente, de los presupuestos que destinan los respectivos gobiernos regionales a la I+D. También hay que tener en cuenta los fondos para investigación procedentes de la Unión Europea y la aportación de las empresas a la investigación en la Universidad. Todo ello incide en una mayor capacidad de autofinanciación de las universidades, que se ha descrito también en el contexto de las universidades europeas (Bonaccorsi y Daraio, 2007).

Para situar el sector de la Enseñanza Superior en España en el conjunto de todos los sectores institucionales, se presenta en la Tabla I el porcentaje que representan los gastos en I+D, el número de investigadores y el porcentaje del gasto en I+D respecto al PIB nacional, en cada uno de los sectores institucionales: Enseñanza Superior; Administración pública (incluye los Organismos Públicos

TABLA I

Distribución de gastos en I+D e investigadores por sectores institucionales

\begin{tabular}{l|c|c|c|c|c|c}
\hline Sectores institucionales & $\begin{array}{c}\text { Gastos } \\
\mathbf{I + D} \\
\text { (miles } € \text { ) }\end{array}$ & $\mathbf{\%}$ & $\begin{array}{c}\text { Número de } \\
\text { investigadores* } \\
\text { EJC }^{* *}\end{array}$ & $\mathbf{\%}$ & $\begin{array}{c}\text { Gastos I+D/ } \\
\text { Investigador } \\
\text { (miles } \boldsymbol{~} \text { ) }\end{array}$ & $\begin{array}{c}\text { \% Gasto } \\
\text { I+D/PIB }\end{array}$ \\
\hline Enseñanza Superior & $\mathbf{2 . 6 4 1 . 6 5 3}$ & $\mathbf{2 9 , 5 2}$ & $\mathbf{5 1 . 5 7 1}$ & $\mathbf{5 1 , 0 6}$ & $\mathbf{5 1 , 2 2}$ & $\mathbf{0 , 3 2}$ \\
Administración Pública & 1.427 .504 & 15,95 & 17.151 & 16,98 & 83,23 & 0,17 \\
Empresa & 4.864 .930 & 54,38 & 32.224 & 31,90 & 150,97 & 0,58 \\
IPSFL & 11.673 & 0,13 & 173 & 0,17 & 67,47 & \\
\hline Todos los sectores & 8.945 .761 & & 100.994 & & 88,58 & 1,07 \\
\hline
\end{tabular}

Fuente: INE, 2004.

* Incluye becarios pre y post-doctorales y contratados.

** EIC. Equivalente a Jornada Completa. 
de Investigación); Empresas; e Instituciones privadas sin fines de lucro (IPSFL), Como se ve en la Tabla I, aunque algo más de la mitad de los investigadores españoles pertenecen a la Universidad, el gasto en I+D de dicho sector corresponde al 30\% del total, mientras que el sector que ejecuta el mayor porcentaje del gasto interno de I+D es el empresarial (54\%).

El estudio de las universidades españolas que se presenta en este artículo tiene en cuenta las comunidades en las que están situadas, no sólo por su directa dependencia administrativa, sino por la influencia del entorno en que se desarrolla la labor de la universidad, a través de las interacciones con el sector Empresa y el sector Administración (Zitt y otros, 2003; Olmeda-Gómez y otros, 2008).

\section{Metodología}

Se analiza la actividad de las universidades españolas a través de indicadores específicos de recursos destinados a la investigación, indicadores estructurales y de docencia, e indicadores bibliométricos que analizan las publicaciones científicas como resultado básico de la investigación. Asimismo, se consideran datos relativos al entorno geográfico en el que se ubican las universidades.

a) Indicadores de desarrollo industrial, relativos a la comunidad autónoma en la que se ubican las universidades:

- PIB de la comunidad autónoma, relativo a la media UE-25.

- Porcentaje del gasto empresarial en I+D de cada comunidad autónoma.

- Porcentaje de población activa en el sector empresa en la región.

b) Indicadores de actividad docente, entre los que se incluyen los siguientes:

- Número de profesores.

- Número de profesores doctores.

- Número de estudiantes.

- Especialización temática de las universidades, medida a través del porcentaje de profesores en el área temática estudiada y el índice de Pratt que mide dispersión de profesores por áreas temáticas.

c) Indicadores estructurales:

- Tipo de universidad: pública o privada.

- Antigüedad de la universidad: años desde su creación.

d) Indicadores bibliométricos o de resultados de investigación

Los datos de resultados se basan en el análisis de las publicaciones científicas de cada universidad durante el período 2001-2005. Se consideran: 1) publicacio- 
nes de orientación internacional, recogidas en el Web of Science (WoS), que incluye el Science Citation Index Expanded (SCIE), Social Sciences Citation Index (SSCI) y Arts \& Humanities Citation Index (A\&HCI); 2) publicaciones de carácter nacional, recogidas en la base de datos ICYT, de Ciencias Experimentales, e ISOC, de Ciencias Sociales y Humanas. Para cada universidad se han calculado los siguientes indicadores:

- Indicadores cuantitativos de actividad científica: número de publicaciones en cada base de datos y porcentaje de documentos en bases de datos nacionales, respecto al total de publicaciones.

- Indicadores de especialización temática:

- A través del índice de Pratt se analiza la dispersión de publicaciones por áreas temáticas (Gómez y otros, 2007), de forma que el índice se incrementa al aumentar la concentración temática. Este índice permite distinguir entre universidades generalistas y especializadas.

- El Índice de Actividad (IA) compara el porcentaje de documentos de una universidad en un determinado tema con el porcentaje correspondiente al total de España en dicho tema (en WoS y en las bases de datos españolas). Un IA superior a la unidad indica que la universidad estudiada dedica al tema en cuestión un mayor porcentaje de sus publicaciones que el promedio del país, lo que conlleva cierto grado de especialización en el tema.

- Indicadores de impacto: número de citas por documento (ventana de citación 2001-2005); porcentaje de documentos no citados; factor de impacto relativo a España (FIR) ${ }^{1}$, citas relativas (número de citas/documento relativo al promedio del país en el tema), porcentaje documentos no citados-relativo al promedio del país en el tema.

- Indicadores de colaboración científica: porcentaje de documentos en colaboración nacional e internacional.

- Indicadores de la transferencia universidad-empresa: porcentaje de documentos en colaboración entre la universidad y el sector industrial.

Este estudio analiza por separado la actividad de las universidades en una selección de áreas temáticas. Con este fin, las disciplinas científicas del WoS se agrupan en nueve grandes áreas (Agricultura-Biología-Medio Ambiente, Biomedicina, Ciencias Sociales, Física, Humanidades, Ingeniería-Tecnología, Matemáticas, Medicina Clínica, y Química). Los datos relativos al profesorado se analizaron también siguiendo esta clasificación, tras adaptar las áreas del conocimiento en las que se distribuye el personal universitario español a las nueve áreas antes mencionadas. El estudio que aquí se presenta se centra en el análisis de cuatro 2004).

1 Se usa el factor de impacto en el año 2004 (fuente: SCI/SSCI Journal Citation Reports 
áreas: 1) Química, 2) Ingeniería/Tecnología, 3) Ciencias Sociales y 4) Ciencias Humanas.

Se analizan los indicadores correspondientes a cada universidad empleando diversas herramientas estadísticas mediante SPSS (versión 13). En los análisis sólo se incluyen las universidades con más de 30 documentos en WoS (Química, Ingeniería) o con más de 30 documentos en WoS o en ISOC (Ciencias Sociales, Ciencias Humanas). Para estudiar la relación entre las variables se utiliza el análisis factorial aplicado al conjunto de universidades públicas, ya que no se dispone de información detallada relativa a las privadas.

\section{Resultados y discusión}

En este trabajo se analizan 67 universidades españolas, 49 públicas y 18 privadas con notables diferencias entre ellas tanto en el número de alumnos y de profesores, como en el gasto en I+D y en el número de publicaciones. En general, la mayoría de las universidades públicas son multidisciplinares, y cuentan con mayor número de cátedras y alumnos que las privadas (el 91\% de alumnos corresponde a las universidades públicas y el $9 \%$ a las privadas). Las universidades politécnicas están dedicadas principalmente a las ramas de ingeniería y tecnología.

En el Anexo I, se presenta la distribución de las universidades públicas y privadas por Comunidades Autónomas, y sus principales indicadores docentes. Las universidades españolas muestran una distribución irregular por regiones, consecuencia del antiguo modelo centralista. Así, en el período estudiado, Madrid contaba con 14 universidades, 6 públicas (entre ellas la Universidad Complutense de Madrid, que es la más grande del país) y 8 privadas, siendo la comunidad autónoma con un mayor número de universidades privadas. Otras comunidades con un alto número de universidades son Cataluña, Andalucía y Valencia, con 11, 9 y 6, respectivamente. Hay que destacar que las nueve universidades de Andalucía son públicas. Las universidades públicas están distribuidas más uniformemente que las privadas por toda la geografía española, y estas últimas tienden a establecerse en las regiones más ricas (Gómez y otros, 2006).

En el período estudiado, el número de profesores en la universidad pública asciende a 37.185. En el Anexo I se observa que el mayor porcentaje de docentes corresponde a Andalucía (19\%), seguido de Madrid (18\%), Cataluña (15\%) y Valencia (10\%), y el menor a la Rioja, con un 0,39\%. No existen estadísticas accesibles y fiables relativas al profesorado de las universidades privadas.

El número total de alumnos, considerando las públicas y las privadas, es de más de 1.300.000, correspondiendo el $91 \%$ a la universidad pública y el $9 \%$ a la privada. Andalucía ocupa el primer lugar en número de alumnos (242.290, lo que supone un 18\%), seguida de Madrid (204.300, 15\%). Es destacable que en Navarra la universidad privada, que cuenta con un gran prestigio, presenta mayor número de alumnos que la pública. 
En la universidad pública el número medio de alumnos por profesor es de 38, oscilando entre 23, en la Universidad de Murcia, y 65 en la de Cantabria. Mención aparte merece la Universidad a Distancia, que por su propia naturaleza, es la que más alumnos por profesor puede abarcar (la media alumnos por profesor es de 197).

La Figura 1 muestra que existe cierta relación entre la antigüedad de las universidades y su nivel de especialización. Así, las universidades más antiguas son generalistas, en cuanto que sus publicaciones tienden a distribuirse por un mayor número de áreas temáticas (la menor concentración se asocia a un menor índice de Pratt). Por su parte, entre las universidades más modernas creadas en el último siglo, se observan distintos grados de especialización, siendo las más especializadas las universidades privadas, tal y como se observa en la parte superior derecha de la Figura 1. Las Escuelas de Ingeniería constituyen un caso particular, ya que a pesar de su gran tamaño y larga historia, son muy especializadas.

\section{FIGURA 1}

Antigüedad y especialización de las universidades públicas y privadas

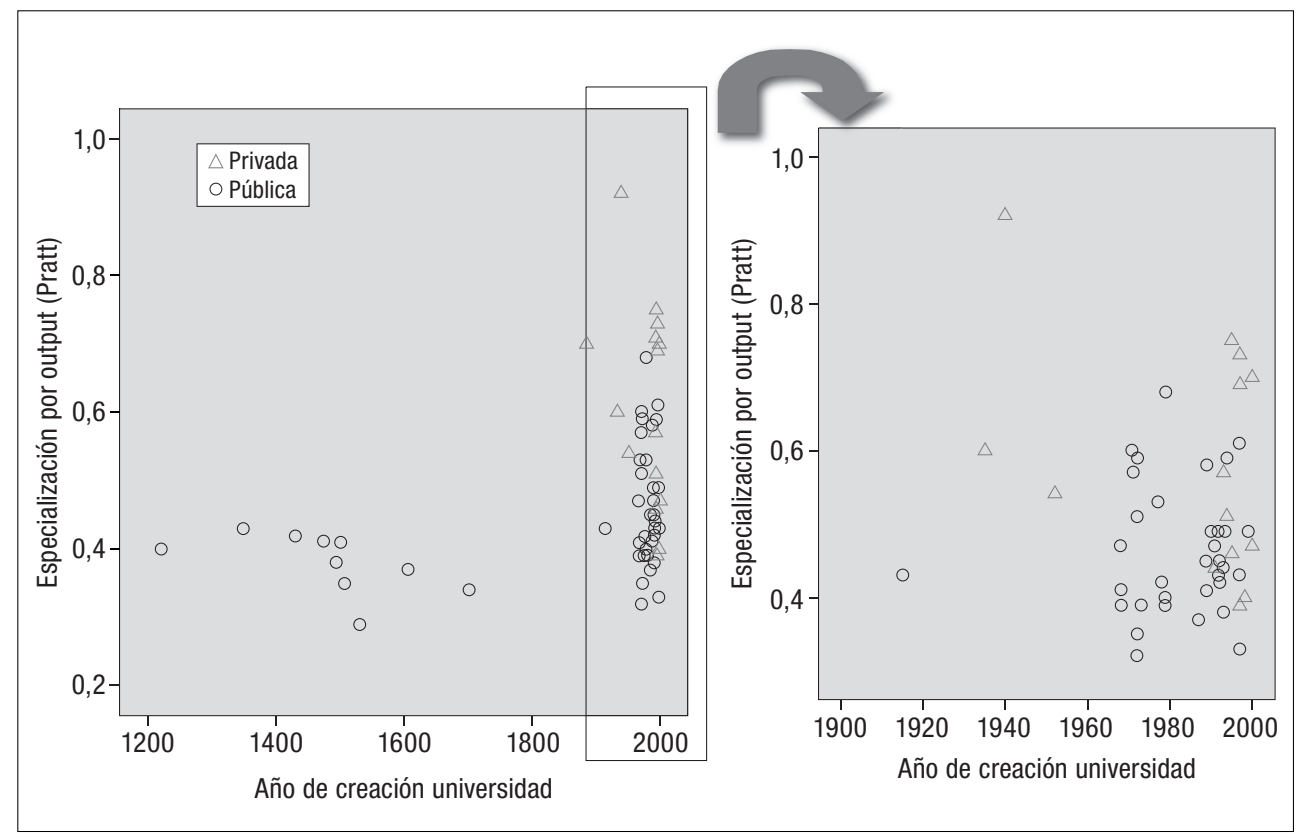

En relación a las publicaciones, ha de señalarse que el sector público en España es el responsable mayoritario de las mismas y, dentro de él, la universidad produce el $62 \%$ de las publicaciones españolas recogidas en la base de datos WoS (Gómez y otros, 2006). En el predominio de la actividad de las universidades públicas influye el hecho de que éstas están más orientadas a la investigación que 
las privadas, donde prima más la enseñanza. Este es también el caso de las Escuelas de Ingeniería, que tradicionalmente han mostrado una mayor dedicación a la docencia y a resolver problemas técnicos para las empresas, que a la investigación.

Es interesante destacar que si bien cerca del $45 \%$ de los profesores universitarios y el $60 \%$ de los alumnos en España pertenecen a las áreas de Ciencias Sociales y Ciencias Humanas, sólo un 9\% de las publicaciones de universidades españolas recogidas en el WoS corresponden a estas áreas. Esta situación se explica en parte por diferencias en la cobertura de las distintas áreas temáticas en la base de datos WoS, que cubre muy bien la Química, de forma moderada las Ingenierías, y de forma incompleta las Ciencias Sociales y, sobre todo, las Humanas (Moed, 2005). La inclusión en este estudio de las publicaciones recogidas en bases de datos españolas (ISOC, para Ciencias Sociales y Ciencias Humanas, e ICYT, en el caso de las Ingenierías) supera parcialmente esta limitación.

\subsection{Diferencias temáticas en los hábitos de publicación y colaboración}

Las diferencias en los hábitos de publicación y colaboración del sector universitario español en las cuatro áreas temáticas seleccionadas se muestran en la Tabla II. Se observa el predominio de las publicaciones internacionales en Química y Tecnología (más del 85\% de sus publicaciones recogidas en WoS), mientras que las publicaciones nacionales adquieren gran importancia en Ciencias

\section{TABLA II}

Producción científica y patrones de colaboración de la universidad española en las áreas temáticas estudiadas (2001-2005)

\begin{tabular}{l|c|c|c|c}
\hline & Química & Tecnología & C. Sociales & C. Humanas \\
\hline N $^{\circ}$ Doc. Wos & 18.284 & 21.138 & 5.556 & 2.602 \\
No Doc. BD española & 326 & 4.023 & 26.927 & 18.849 \\
\% Doc. BD española/total doc. & 1,75 & 15,99 & 82,90 & 87,87 \\
\hline WoS & & & & \\
\% Colaboración nacional & 39,5 & 38 & 33,1 & 6,2 \\
\% Colaboración internacional & 32,7 & 32 & 27,9 & 5,7 \\
\% No colaboración & 37,3 & 38,4 & 46,4 & 89,0 \\
\% Colaboración empresa & 2,18 & 2,52 & 1,31 & 0,15 \\
\hline BD nacionales & & & & \\
\% Colaboración nacional & 23,01 & 28,49 & 11,89 & 5,96 \\
\% Colaboración internacional & 7,98 & 6,64 & 2,24 & 1,34 \\
\% No colaboración & 70,55 & 66,27 & 86,26 & 92,97 \\
\% Colaboración empresa & 3,99 & 8,75 & 0,41 & 0,26 \\
\hline
\end{tabular}

Nota: Las BD españolas son ICYT —en Química y Tecnología—, e ISOC —en Ciencias Sociales y Humanas- 
Sociales (83\%) y, especialmente, en Ciencias Humanas (88\%).

En las áreas de Ciencias Sociales y Ciencias Humanas menos del 20\% de los documentos del sector universitario se difunden a través de artículos en revistas internacionales (WoS). Por esta razón, en el estudio de estas áreas tiene especial interés analizar las publicaciones nacionales recogidas en ISOC, además de las incluidas en WoS. En el caso de Química e Ingeniería las publicaciones nacionales son minoritarias, pero también se han incluido mediante consulta de la base de datos ICYT.

\subsection{Análisis multifactorial de la actividad de las universidades}

Partiendo del amplio número de variables mostradas en la metodología, en este trabajo se utiliza el análisis factorial para identificar los principales factores subyacentes que clarifican la relación entre las variables correspondientes a las universidades públicas en cada una de las cuatro áreas objeto de análisis.

Debido a las peculiaridades de las Ciencias Sociales y Humanas, las variables incluidas en su análisis multivariante difieren ligeramente de las correspondientes a Química y Tecnología, pues se consideran los valores absolutos de producción en WoS e ISOC, y el PIB regional, pero no se tienen en cuenta todos los indicadores directamente relacionados con el sector Empresa, por la escasa incidencia de este sector en dichas áreas.

Por otro lado, la interpretación de los resultados relativos a las publicaciones de difusión internacional en las Ciencias Sociales y Humanas debe hacerse con cautela, por la escasa presencia de este tipo de publicaciones en estas áreas y la menor validez de las citas en las disciplinas humanísticas.

\section{a) Área temática de Química}

Como se observa en la Tabla II, las publicaciones de Química en la base de datos española ICYT representan menos del 2\% del total, se trata de un área muy internacionalizada. El análisis factorial permite obtener cuatro factores que explican el $71 \%$ de la varianza (Tabla III).

El primer factor agrupa las variables relativas a desarrollo industrial: las regiones ricas con elevado PIB, con alta participación del sector industrial en las inversiones en I+D y elevado porcentaje de población ocupada en el sector industrial, tienden a mostrar una mayor colaboración universidad-empresa.

El segundo factor está relacionado con el tamaño y antigüedad de las universidades: el número de profesores y de publicaciones WoS se relaciona positivamente con la antigüedad de la universidad, y negativamente con la colaboración nacional, y con la proporción de documentos no citados.

El tercer factor recoge variables relacionadas con la visibilidad internacional de las publicaciones: las citas recibidas, el factor de impacto relativo, y la cola- 
boración internacional en WoS se relacionan inversamente con la proporción de documentos no citados y el peso de las publicaciones nacionales recogidas en la base de datos ICYT.

Finalmente, el cuarto factor se relaciona con la especialización de la universidad, medida tanto a través del porcentaje de profesorado en Química como de la actividad relativa de la universidad en el área (Índice de Actividad, IA), y correlaciona positivamente con el factor de impacto relativo (FIR).

TABLA III

Química. Matriz de componentes principales rotados

\begin{tabular}{|c|c|c|c|c|}
\hline & \multicolumn{4}{|c|}{ Componentes } \\
\hline & $\begin{array}{c}1 \\
\text { Riqueza } \\
\text { industrial }\end{array}$ & $\begin{array}{c}2 \\
\text { Tamaño y } \\
\text { antigüedad }\end{array}$ & $\begin{array}{c}3 \\
\text { Visibilidad } \\
\text { internacional }\end{array}$ & $\begin{array}{c}4 \\
\text { Especiali- } \\
\text { zación }\end{array}$ \\
\hline Gasto I+D empresa & 0,891 & & & \\
\hline \% Población activa empresa & 0,788 & & & 0,337 \\
\hline PIB_CCAA & 0,686 & & & $-0,360$ \\
\hline \% Colaboración nac. WoS & 0,662 & $-0,492$ & & \\
\hline \% Colaboración empresa WoS & 0,536 & & & \\
\hline No Prof. Química & & 0,906 & & \\
\hline Antigüedad & & 0,878 & & \\
\hline $\mathrm{N}^{\mathrm{o}}$ Doc. WoS & & 0,849 & & \\
\hline \% Colaboración internac. WoS & & & 0,790 & \\
\hline Citas relativas & & & 0,734 & \\
\hline FIR & & & 0,663 & 0,414 \\
\hline$\%$ No citados-relat. & $-0,341$ & $-0,402$ & $-0,610$ & \\
\hline \% Doc. ICYT/total doc. & & & $-0,603$ & \\
\hline Índice Actividad WoS & & & & 0,902 \\
\hline \% Prof. Química & & & & 0,820 \\
\hline
\end{tabular}

Método de extracción: Análisis de componentes principales.

Método de rotación: Normalización Varimax con Kaiser.

La rotación ha convergido en 6 iteraciones.

Nota: solo se muestran cargas factoriales $>0,3$

La Figura 2 muestra en el eje vertical la riqueza y el desarrollo industrial de las regiones donde están ubicadas las universidades, y presenta en el eje horizontal la especialización en Química. Destacan por su situación en regiones económicamente ricas y por su alta especialización en el área algunas universidades como la de La Rioja o la Universidad Rovira i Virgili (URIV). La Universidad de 
Burgos, a la derecha de la figura, es la que muestra mayor especialización en el área (más de la mitad de su producción en WoS corresponde al área de Química), mientras que la Universidad de Lérida se sitúa en la parte superior de la figura por su localización en una comunidad autónoma rica (Cataluña), y presentar altos valores de colaboración con la empresa en WoS .

\section{FIGURA 2}

Química. Relación entre el factor 1 (desarrollo industrial de la Comunidad Autónoma) y el factor 4 (especialización en Química)

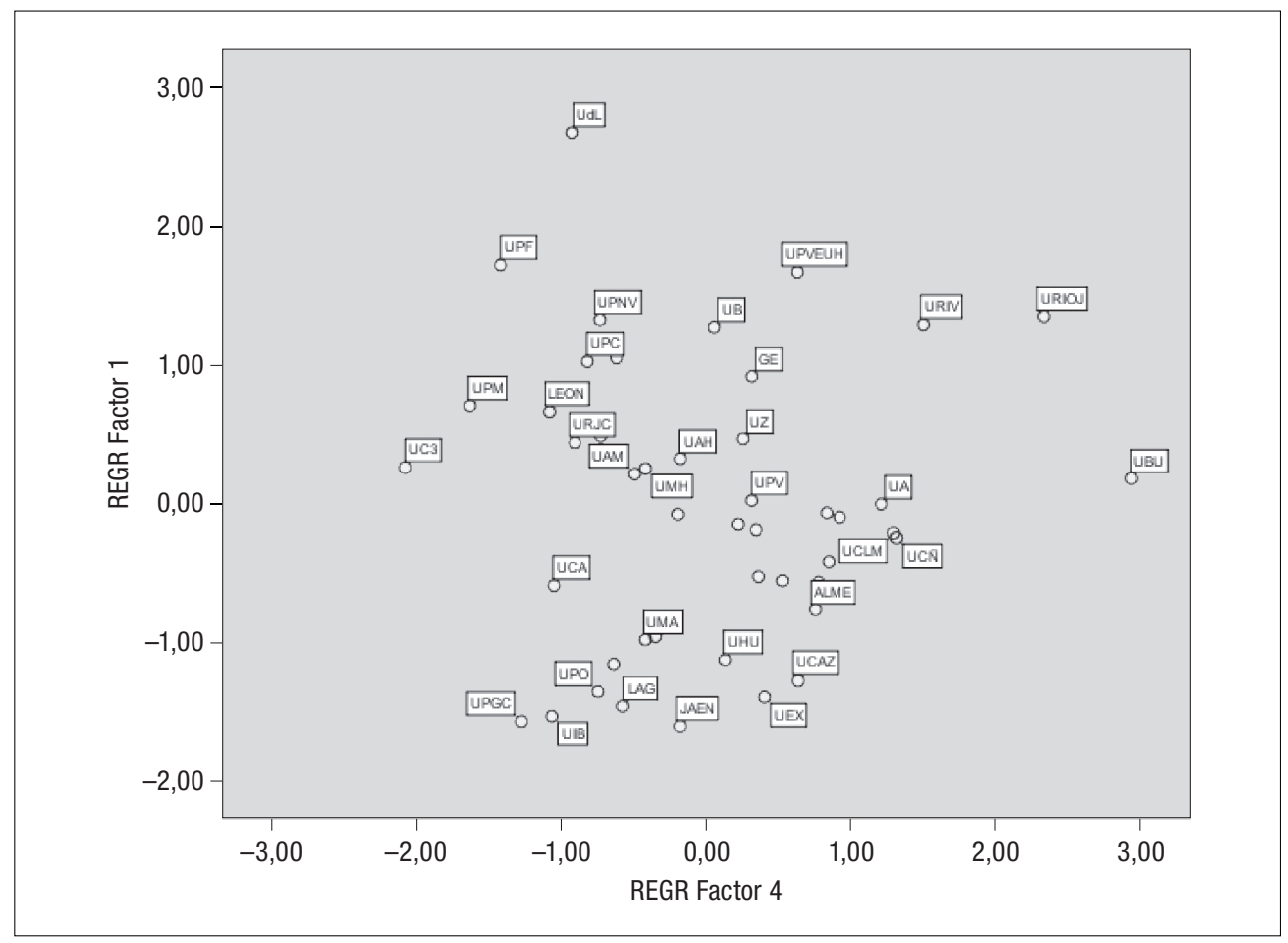

Nota: abreviaturas de las universidades en el Anexo II.

\section{b) Área temática de Ingeniería-Tecnología}

En el área de Ingeniería-Tecnología la producción científica internacional WoS es muy superior (84\%) a la recogida por la base de datos ICYT, que supone el $16 \%$ (Tabla II). A través del análisis factorial, se obtienen cinco factores, que explican el $81 \%$ de la varianza.

Según la Tabla IV, en esta área se mantienen los factores relativos a la visibilidad internacional, entorno de desarrollo industrial y tamaño, ya descritos en 
el área anterior, y se añaden dos nuevos factores. Es interesante observar que en este caso el tamaño de las universidades (número de profesores) se incrementa con la especialización (\% profesores en el área, índice de actividad) (factor 1), lo que se explica en el contexto de la importancia de las universidades politécnicas en el área. Por otro lado, es llamativo que la colaboración internacional en las publicaciones WoS contribuye a tres factores: facilita la obtención de alto impacto (factor 2), pero también se ve propiciada por un desarrollo industrial rico (factor 3), y es característica de algunas universidades de larga historia (factor 4). Hay que señalar que la colaboración con la empresa tiende a incrementarse en las regiones con mayor PIB, en las que también tiende a producirse una mayor colaboración internacional, pero también se asocia a universidades de mayor antigüedad, en las que predomina la orientación local de las publicaciones (factor 5).

TABLA IV

Ingeniería-Tecnología. Matriz de componentes principales rotados

\begin{tabular}{|c|c|c|c|c|c|}
\hline & \multicolumn{5}{|c|}{ Componentes } \\
\hline & $\begin{array}{c}1 \\
\text { Tamaño y } \\
\text { especializ. }\end{array}$ & $\begin{array}{c}2 \\
\text { Visibilidad }\end{array}$ & $\begin{array}{c}3 \\
\text { Desarrollo } \\
\text { industrial }\end{array}$ & $\begin{array}{c}4 \\
\text { Colab. int. y } \\
\text { antigüedad }\end{array}$ & $\begin{array}{c}5 \\
\text { Colab. } \\
\text { empresa }\end{array}$ \\
\hline $\mathrm{N}^{\mathrm{o}}$ Prof. Ingeniería & 0,921 & & & & \\
\hline$\%$ Prof. Ingeniería & 0,888 & & & & \\
\hline Índice Actividad WoS & 0,841 & & & & \\
\hline $\mathrm{N}^{\mathrm{o}}$ Doc WoS & 0,840 & & & & \\
\hline Citas relativas & & 0,925 & & & \\
\hline No citados-relat. & & $-0,871$ & & & \\
\hline FIR & & 0,865 & & & \\
\hline Gasto I+D empresa & & & 0,909 & & \\
\hline$\%$ Poblac. activa empresa & & & 0,780 & & \\
\hline PIB_CCAA & & & 0,756 & 0,303 & \\
\hline \% Col. Nac. WoS & & & & $-0,846$ & \\
\hline$\%$ Col. Int. WoS & & 0,315 & 0,442 & 0,702 & \\
\hline \% Doc. ICYT/tot. doc. & & & & & 0,779 \\
\hline Antigüedad & & 0,377 & & 0,521 & 0,620 \\
\hline \% Col. Empr. WoS & & & 0,478 & & 0,552 \\
\hline
\end{tabular}

Método de extracción: Análisis de componentes principales.

Método de rotación: Normalización Varimax con Kaiser.

La rotación ha convergido en 7 iteraciones.

Nota: solo se muestran cargas factoriales $>0,3$ 
En la Figura 3 destacan la Universidad Politécnica de Madrid (UPM) y la de Cataluña (UPC) por su alta especialización en el área (factor 1), mientras que en términos de visibilidad internacional (factor 2) sobresalen la Universidad de Barcelona (UB) y la de Córdoba (UCOR) a la derecha.

FIGURA 3

Ingeniería. Relación entre el factor 1 (tamaño y especialización de las universidades) y el factor 2 (visibilidad internacional)

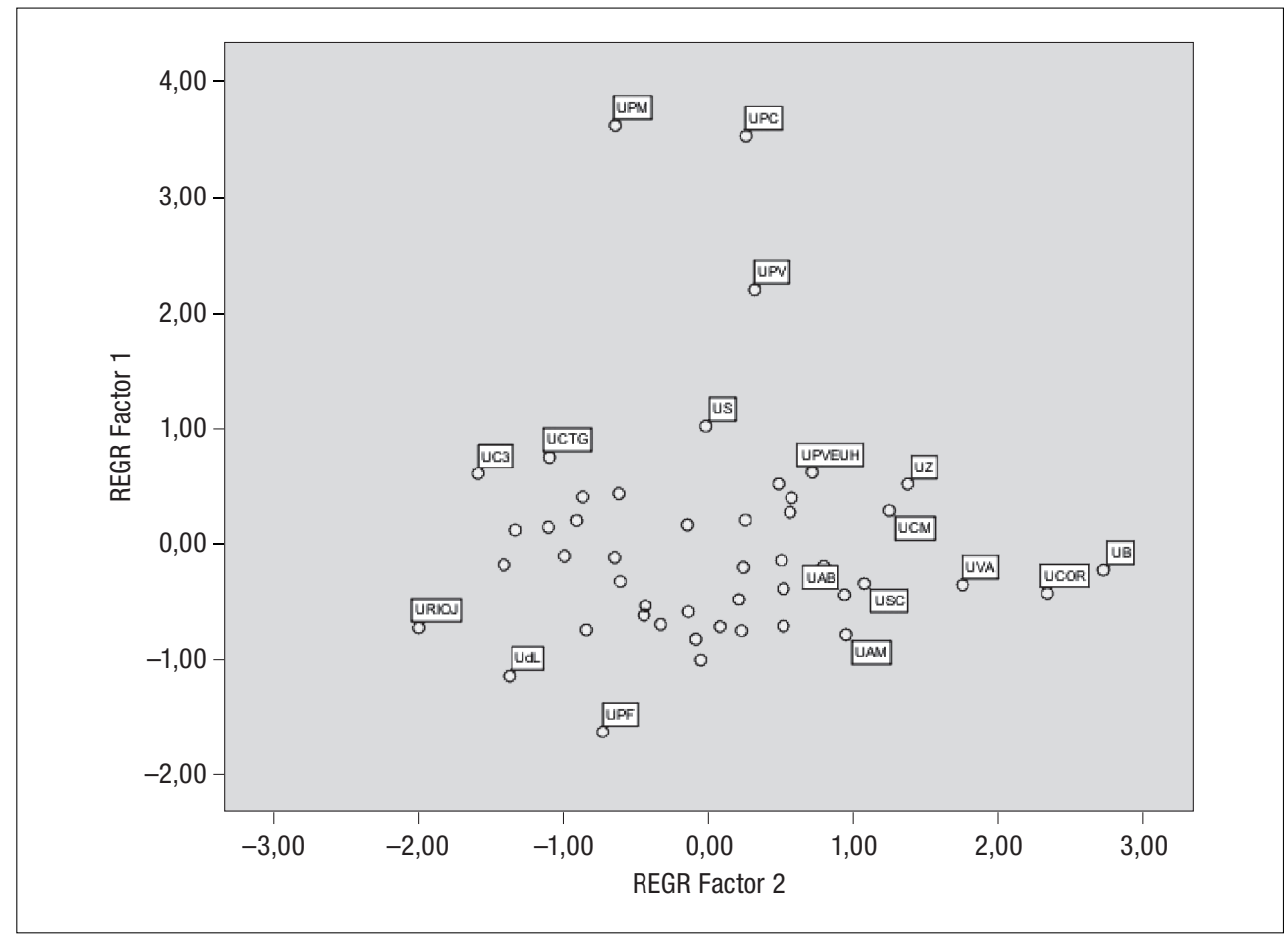

Nota: abreviaturas de las universidades en el Anexo II.

La representación del factor 1 (especialización) frente al factor 3 (desarrolo industrial) permite distinguir una serie de universidades politécnicas en la parte superior de la Figura 4, mientras que sobresalen por la riqueza industrial de sus regiones y su colaboración con la industria las Universidades del País Vasco (UPVEUH), la Rioja (URIOJ), Lérida (UdL) y Universidad Pompeu Fabra (UPF). 


\section{FIGURA 4}

Ingeniería. Relación entre el factor 1 (tamaño y especialización) y el factor 3 (desarrollo regional)

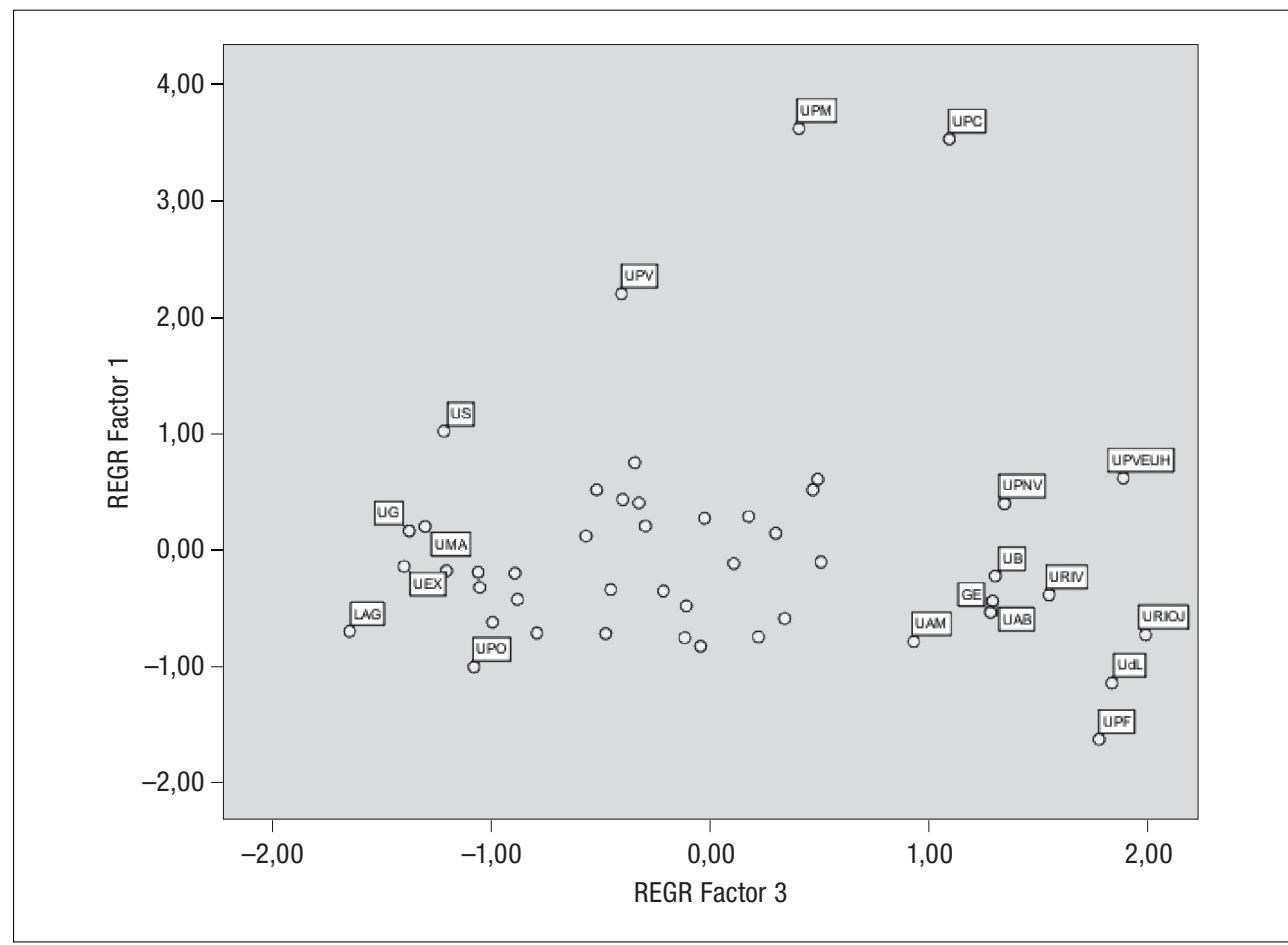

Nota: abreviaturas de las universidades en el Anexo II

\section{c) Área temática de Ciencias Sociales}

La producción científica en Ciencias Sociales está infra-representada en WoS, ya que solo recoge el $17 \%$ de las publicaciones del área. En cuanto a disciplinas, la más productiva es la Economía, tanto en la base internacional WoS como en la española ISOC.

A través del análisis factorial se obtienen cinco factores, que explican el 79\% de la varianza (Tabla V). El factor 1 se refiere al tamaño de las universidades, observándose en este caso que las de mayor tamaño (mayor numero de profesores) son las de mayor antigüedad, y tienen más producción nacional e internacional. Al factor 2 contribuyen las citas y la colaboración internacional en las publicaciones WoS. El factor 3 se refiere a la especialización temática de las universidades, mostrándose en este caso una correlación positiva entre especialización medida por publicaciones (tanto ISOC como WoS) y por el área del conocimiento del profesorado. La mayor especialización se muestra en las uni- 
versidades más jóvenes. El factor 4 incluye los datos relativos al desarrollo industrial, que en este caso no parecen influir de forma importante y diferencial sobre la actividad de las universidades. Curiosamente, el incremento de la colaboración nacional en WoS se asocia a la publicación en revistas de mayor factor de impacto, y se opone a la antigüedad de las universidades (factor 5).

\section{TABLA V}

Ciencias Sociales. Matriz de componentes principales rotados

\begin{tabular}{|c|c|c|c|c|c|}
\hline & \multicolumn{5}{|c|}{ Componentes } \\
\hline & $\begin{array}{c}1 \\
\text { Tamaño y } \\
\text { antigüedad }\end{array}$ & $\begin{array}{c}2 \\
\text { Visibilidad } \\
\text { internacional }\end{array}$ & $\begin{array}{c}3 \\
\text { Especializa- } \\
\text { ción }\end{array}$ & $\begin{array}{c}4 \\
\text { Desarrollo } \\
\text { industrial }\end{array}$ & $\begin{array}{c}5 \\
\text { Impacto }\end{array}$ \\
\hline$N^{o}$ Prof. Sociales & 0,967 & & & & \\
\hline $\mathrm{N}^{\mathrm{o}}$ Doc. ISOC & 0,957 & & & & \\
\hline $\mathrm{N}^{\mathrm{o}}$ Doc. WoS & 0,845 & 0,325 & & & \\
\hline Antigüedad & 0,531 & & $-0,410$ & & $-0,424$ \\
\hline \% Col. Nac. WoS & $-0,493$ & $-0,484$ & & & 0,444 \\
\hline Citas relativas & & 0,840 & & & \\
\hline No citados-relat. & & $-0,840$ & & & \\
\hline \% ISOC/Total doc. & & $-0,575$ & $-0,498$ & $-0,382$ & \\
\hline \% Col. Int. WoS & & 0,538 & 0,437 & & \\
\hline Índice Actividad WoS & & & 0,926 & & \\
\hline \% Prof. Sociales & & & 0,883 & & \\
\hline Gasto I+D empresa & & & & 0,930 & \\
\hline$\%$ Poblac. activa industria & & & & 0,805 & \\
\hline PIB_CCAA & & & & 0,746 & \\
\hline FIR & & 0,309 & & & 0,797 \\
\hline
\end{tabular}

Método de extracción: Análisis de componentes principales.

Método de rotación: Normalización Varimax con Kaiser.

La rotación ha convergido en 5 iteraciones.

Nota: solo se muestran cargas factoriales $>0,3$

En la Figura 5 se observa que la Universidad Complutense de Madrid (UCM), es la más grande y generalista, mientras que la Universidad Pompeu i Fabra (UPF) destaca por su alta especialización. 


\section{FIGURA 5}

Ciencias Sociales. Relación entre el factor 1 (tamaño y antigüedad) y el factor 3 (especialización temática)

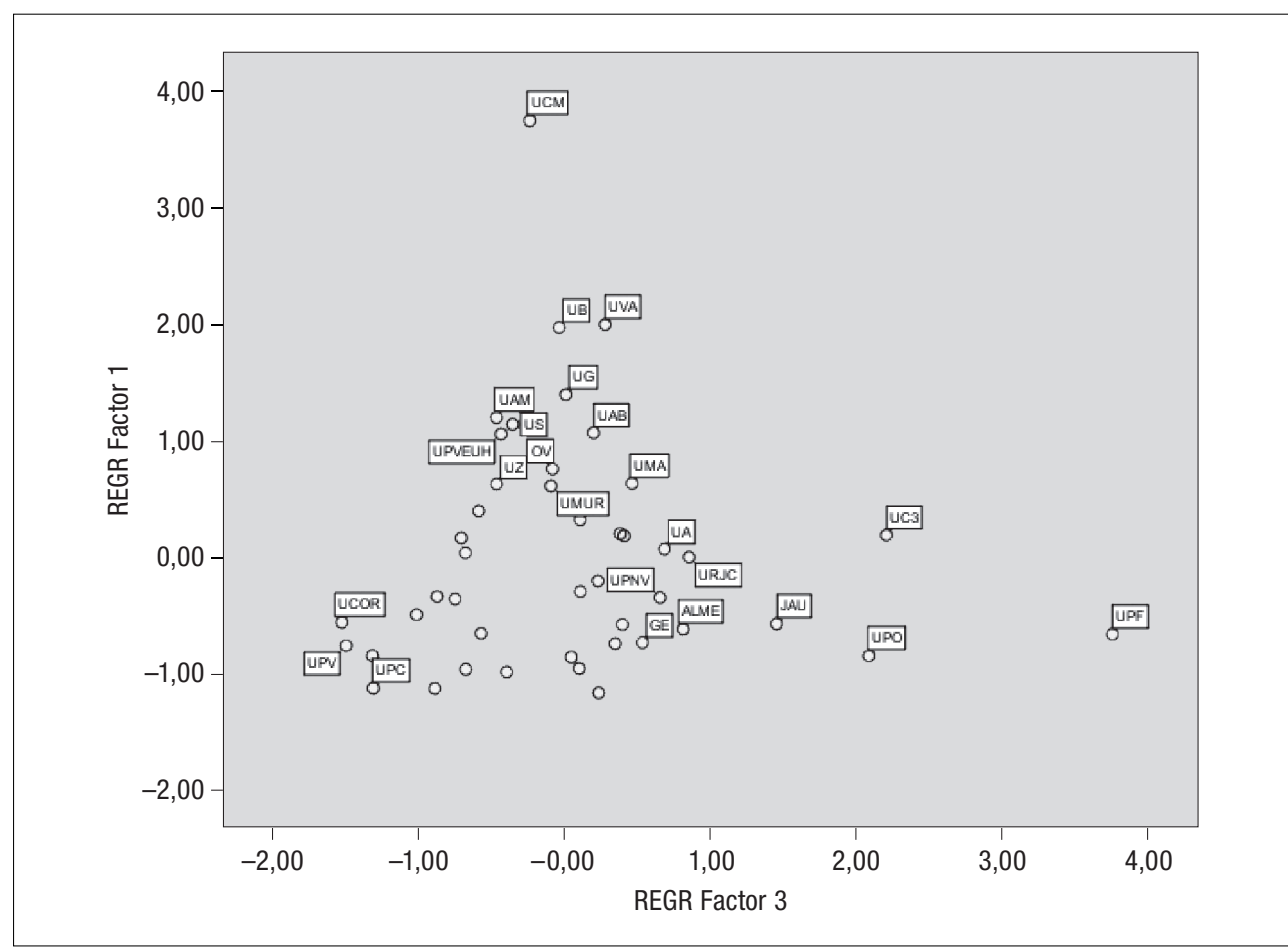

Nota: abreviaturas de las universidades en el Anexo II

\section{d) Área temática de Ciencias Humanas}

En esta área tienen un importante peso las publicaciones nacionales, de forma que el $88 \%$ de los artículos se recogen en la base de datos ISOC. No obstante, hay que señalar que esta base de datos no analiza libros ni monografías, que son otros tipos documentales muy relevantes en las Ciencias Humanas. El análisis factorial proporciona cinco factores, que explican el $86 \%$ de la varianza.

Los resultados en esta área (Tabla VI) son similares a los descritos en el área anterior. Por un lado, las publicaciones WoS e ISOC tienden a aumentar junto con el número de profesores y la antigüedad del centro (factor 1). El impacto, medido por las citas recibidas, se incrementa con la colaboración internacional, aunque hay que tener en cuenta que las citas son un indicador de menor validez en esta área (factor 2). En lo que se refiere a la especialización, se observa que coexisten dos tipos de universidades: algunas de gran tamaño, más especializadas 
en publicaciones internacionales (factor 3), y otras en las que converge la especialización nacional, internacional y por profesorado (factor 4). La riqueza regional no parece influir de forma decisiva y diferencial sobre la actividad universitaria y permanece aislada (factor 5).

TABLA VI

Ciencias Humanas. Matriz de componentes principales rotados

\begin{tabular}{|c|c|c|c|c|c|}
\hline & \multicolumn{5}{|c|}{ Componente } \\
\hline & $\begin{array}{c}1 \\
\text { Tamaño y } \\
\text { antigüedad }\end{array}$ & $\begin{array}{c}2 \\
\text { Visibilidad } \\
\text { internac. }\end{array}$ & $\begin{array}{c}3 \\
\text { Orientación } \\
\text { internac. }\end{array}$ & $\begin{array}{c}4 \\
\text { Especiali- } \\
\text { zación }\end{array}$ & $\begin{array}{c}5 \\
\text { Riqueza } \\
\text { regional }\end{array}$ \\
\hline $\mathrm{N}^{\circ}$ Prof. C. Humanas & 0,950 & & & & \\
\hline $\mathrm{N}^{\circ}$ Doc. ISOC & 0,943 & & & & \\
\hline $\mathrm{N}^{\mathrm{o}}$ Doc. WoS & 0,874 & & & & \\
\hline Antigüedad & 0,827 & & & & \\
\hline No citados-relat. & & $-0,914$ & & & \\
\hline Citas relativas & & 0,912 & & & \\
\hline \% Col. Int. WoS & & 0,751 & & & \\
\hline \% Doc. ISOC/Total Doc. & & & $-0,925$ & & \\
\hline Índice Actividad WoS & & & 0,804 & 0,430 & \\
\hline \% Prof. C. Humanas & & & & 0,914 & \\
\hline PIB_CCAA & & & & & 0,934 \\
\hline
\end{tabular}

Método de extracción: Análisis de componentes principales.

Método de rotación: Normalización Varimax con Kaiser.

La rotación ha convergido en 5 iteraciones.

Nota: solo se muestran cargas factoriales $>0,3$ 


\section{FIGURA 6}

Ciencias Humanas. Relación entre el factor 1 (tamaño y antigüedad) y el factor 4 (especialización temática)

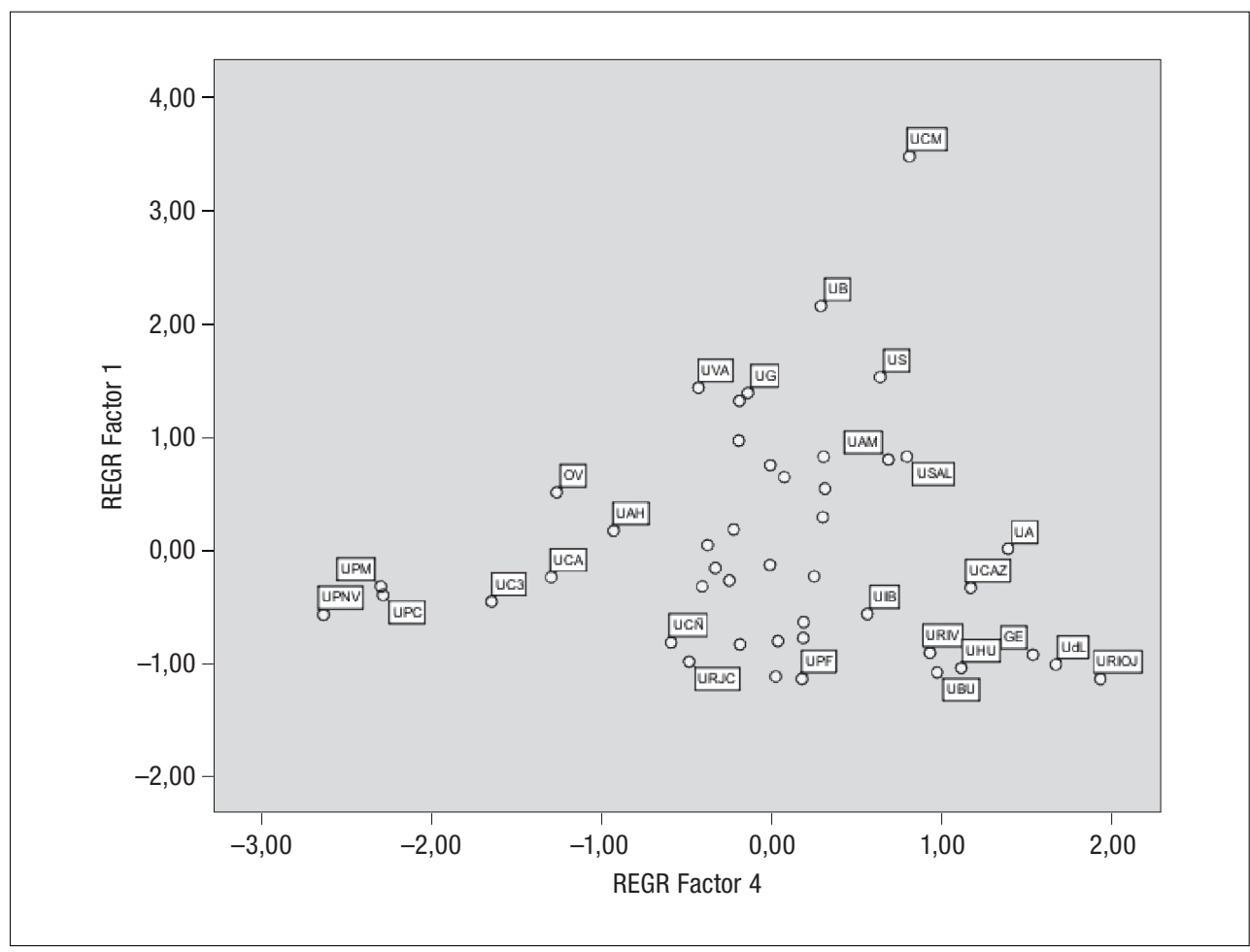

Nota: abreviaturas de las universidades en el Anexo II.

\section{Conclusiones}

Es un hecho admitido que los estudios comparativos de la actividad científica de distintas instituciones se ven limitados por diferencias en la misión, financiación y especialización temática de las mismas (Thijs y Glänzel, 2008). En relación con esta última característica, en este estudio se ponen de manifiesto los distintos hábitos de publicación y colaboración científica según las áreas temáticas, que desaconsejan el estudio global de las universidades, por la heterogeneidad derivada de las diferencias en su perfil temático de actividad. En este sentido, son claras las diferencias entre un área de alta orientación internacional como la Química, y otras áreas como las Ciencias Sociales y Humanas en las que predominan las publicaciones españolas y la colaboración juega un papel muy reducido. Estas diferencias señalan la necesidad de realizar estudios desagregados por áreas, que es lo que se ha pretendido en este trabajo. 
El estudio de la interacción entre las distintas variables permite observar que algunos comportamientos son comunes a las diferentes áreas. Así, el papel positivo de la colaboración internacional sobre la visibilidad internacional (citas) de las publicaciones se verifica en las cuatro áreas analizadas, incluidas las Ciencias Humanas aunque en ella los indicadores basados en citas WoS son menos fiables (Moed, 2005) y el porcentaje de documentos internacionales es muy reducido. La mayor influencia de la investigación realizada en colaboración internacional ha sido descrita en distintas áreas y por diferentes autores (Narin et al., 1991; Katz y Hicks, 1997; Glänzel, 2001; Persson, Glänzel y Danell, 2004). Aunque algunos estudios la han relacionado con una mayor tasa de autocitación derivada del mayor número de autores que generalmente se asocia a la colaboración internacional (Herbertz, 1995), otros trabajos han puesto de manifiesto que el incremento de citación se obtiene principalmente a través de citas externas (Glänzel y Thijs, 2004; Costas y otros, 2009).

Un hallazgo interesante es el relativo a los factores subyacentes a la colaboración entre la universidad y el sector empresa, ya que este vínculo se considera esencial actualmente para facilitar la transferencia de conocimiento desde el sector académico hacia el sector productivo. La colaboración universidad-empresa se encuadra dentro de la llamada "tercera misión" de la universidad, que es la de facilitar el flujo de conocimientos y tecnología hacia la industria y la sociedad, y que se añade a las misiones tradicionales de la universidad: la docencia y la investigación.

Nuestros datos muestran que, en Química y Tecnología, la riqueza y la industrialización de las regiones, medida a través del PIB de la Comunidad Autónoma o de la actividad investigadora financiada por el sector privado, se asocia a una mayor colaboración de la universidad con dicho sector. Estudios previos han descrito el efecto positivo sobre las universidades de poseer un entorno industrial rico, por el desarrollo de sinergias entre ambos tipos institucionales (Zitt y otros, 2003). En nuestro estudio esta relación no se verifica en las áreas de Ciencias Sociales y Humanas, probablemente porque existe menor actividad empresarial que pueda beneficiarse de la investigación académica en estas áreas. Esto no implica que la universidad no contribuya al desarrollo de su tercera misión en estas áreas, sino que lo hace a través de otros mecanismos (asesoramiento gubernamental, divulgación científica, etc.) que no se detectan a través de los indicadores utilizados.

La colaboración universidad-empresa es reducida en el contexto español, asciende al 2\% de las publicaciones WoS de la universidad, y al $4 \%$ en el caso de las publicaciones en revistas españolas (Gómez y otros, 2009). En un estudio previo que analizaba las universidades de forma global, sin diferenciar por áreas temáticas, se observó cierta asociación positiva entre colaboración universidadempresa, riqueza industrial de la región y especialización universitaria, relacionadas inversamente con la edad de la universidad (Gómez y otros, 2009). En el estudio actual diferenciado por áreas, se observa que en las Ingenierías la colaboración universidad-empresa puede desarrollarse en dos entornos diferentes: en 
zonas ricas, asociado a colaboración internacional y, por tanto, a intereses supranacionales; y en universidades muy consolidadas, asociado a intereses locales como se infiere de un alto porcentaje de publicaciones ICYT.

Diversos estudios señalan que la diferenciación de las universidades es una respuesta estratégica frente a un ambiente de creciente competitividad. Así, es posible la diferenciación horizontal, que supone la especialización de la universidad bien hacia la dimensional profesional o bien hacia la académica; o la diferenciación vertical, que distingue entre universidades generalistas o especializadas (Bonaccorsi y Daraio, 2007). Según Bonaccorsi y Daraio (2007), la diferenciación de las universidades europeas es mucho menor que la de las norteamericanas y, entre las europeas, España y otros países mediterráneos presentan la menor diferenciación. Nuestro estudio muestra que las universidades antiguas son generalistas y de mayor tamaño, porque han ido creciendo para cubrir mayor número de disciplinas. Por el contrario, la mayor especialización se encuentra en las universidades politécnicas y en las de reciente creación.

¿Es la especialización de las universidades un factor importante en la visibilidad e influencia de sus resultados de investigación? ¿Es un factor determinante del buen hacer docente e investigador? Un estudio reciente sobre el conjunto de universidades más productivas en el Web of Science (Moed, 2006) permitió observar que el impacto de las universidades en sus áreas de mayor especialización era mayor que el correspondiente a dichas áreas en las universidades generalistas sólo en cuatro disciplinas (ciencias biológicas, medicina clínica, biología molecular/bioquímica y física) de un total de quince consideradas. Se constata en dicho estudio que las cuatro disciplinas mencionadas presentaban cierto componente de "big science", lo que podría explicar la mayor relevancia del concepto de "masa crítica" en las mismas. No obstante, hay que señalar que el estudio señalado se refiere a una selección de universidades de todo el mundo, y que el comportamiento de las universidades puede variar en función de los países y del tipo de universidad.

En nuestro estudio, la especialización de la universidad parece positiva en el área de Química, ya que se asocia a la publicación en revistas de mayor factor de impacto, observándose que las citas se incrementan con la orientación internacional de las publicaciones (menor porcentaje de documentos ICYT). En el caso de las Ingenierías, el prestigio de las revistas de publicación tiende a incrementarse con la edad de las universidades, y no parece aumentar con la especialización. La mayor especialización en Ingeniería corresponde a las universidades politécnicas, pero algunas universidades generalistas obtienen mayor visibilidad internacional en el área y sería interesante explorar si ello se asocia a un perfil temático diferente. En el área de Ciencias Sociales, las universidades más jóvenes son las que muestran mayor especialización, y mayor orientación a la colaboración internacional (ver factor 3 de la Tabla V), pero el impacto tiende a aumentar con el volumen de producción WoS (ver factor 2 de la Tabla V), lo que señala nuevamente la importancia de alcanzar cierta masa crítica de producción para alcanzar las mejores revistas y obtener un retorno en forma de citas. 
En relación a la metodología de este estudio hay que señalar que el output analizado en este trabajo corresponde sólo a las publicaciones en revistas científicas. Sería interesante incluir en futuros análisis otros tipos documentales que tienen especial relevancia en determinadas áreas como son los informes y patentes en Ingeniería, o los libros y monografías en las Ciencias Sociales y Humanas. El interés de estos tipos documentales se ha descrito tanto en la literatura internacional (Hicks, 2004) como en estudios concretos relativos a nuestro país (Moros y Bordons, 2003; Gómez y otros, 2009; Torres-Salinas y otros, 2009).

En resumen, este trabajo pone de manifiesto el interés de considerar el perfil temático de las universidades en el estudio de su actividad, tanto para profundizar en la interacción entre variables estructurales, funcionales y de contexto, como para identificar los principales determinantes de éxito docente y/o investigador.

\section{Agradecimientos}

Esta investigación se ha desarrollado dentro del Proyecto SEJ2004-08052-C02-02/ SOCI, financiado por el Plan Nacional. Una versión preliminar de este trabajo fue presentada en el Seminario Internacional de Estudios Cuantitativos y Cualitativos de Ciencia y Tecnología. INFO2008 (Cuba).

\section{Referencias}

Bonaccorsi, A., y Daraio, C. (2007): Universities and strategic knowledge creation. Specialisation and performance in Europe. Edward Elgar Publishing Limited: Cheltemham, UK.

Bonaccorsi, A.; Daraio, C. y Simar, L. (2006): Advanced indicators of productivity of universities. An application of robust nonparametric methods to Italian data. Scientometrics, 66 (2): 389-410

Costas, R.; Van Leeuwen, T. N., y Bordons, M. (2010): Self-citations at the meso and individual levels: effects of different calculations methods. Scientometrics (en prensa).

De Miguel, J.; Caïs, J., y Vaquera, E. (2001): Excelencia. Calidad de las universidades españolas. Centro de Investigaciones Sociológicas, Madrid.

Glänzel, W. (2001): National characteristics in international scientific co-authorship relations. Scientometrics, 51 (1): 69-115.

Glänzel, W., y Schubert, A. (2001): Double effort=Double impact? A critical view at international co-authorship in chemistry. Scientometrics, 50 (2): 199-214.

Glänzel, W., y Thijs, B. (2004): Does co-authorship inflate the share of self-citations? Scientometrics, 61 (3): 395-404.

Gómez, I.; Bordons, M.; Fernández, M. T., y Morillo, F. (2009): Structure and research performance of Spanish universities. Scientometrics, vol. 79 (1), 131-146.

Gómez, I.; Sancho, R.; Bordons, M., y Fernández, M. T. (2006): La I+D en España a través de publicaciones y patentes. En Sebastián, J. y Muñoz, E. (eds.): Radiografía de la Investigación pública en España. Biblioteca Nueva. Madrid, 273-301. 
Gómez, I.; Bordons, M.; Fernández, T.; Morillo, F., y Costas, R. (2009): Dinámica de las organizaciones de investigación: una aproximación bibliométrica. Informe final proyecto 2004-08052-C02-02/SOCI. IEDCYT-CCHS, CSIC. Madrid, 2009.

Gómez, I.; Bordons, M.; Morillo, F.; Aparicio, J.; Candelario, A., y Herrero, M. (2009): La actividad científica del CSIC a través del Web of Science. Estudio bibliométrico del período 2000-2007. IEDCYT-CCHS, CSIC. Madrid.

Herbertz, H., y Müller-Hill, B. (1995): Quality and efficiency of basic research in molecular biology: a bibliometric analysis of thirteen excellent research institutes. Research Policy, 24, 959-979.

Hicks, D. (2004): The four literatures of Social Science. En Moed, H. F.; Glänzel, W. y Schmoch, U. (eds.): Handbook of Quantitative Science and Technology Research. Kluwer Academic Publishers. The Netherlands, 473-498.

INE. Instituto Nacional de Estadística. http://ine.es/inebase.

Katz, J. S., y Hicks, D. (1997): How much is collaboration worth? A calibrated bibliometric model. Scientometrics, 40 (3), 541-554.

Larivière, V.; Gingras, Y., y Archambault, E. (2006): Canadian collaboration networks: A comparative analysis of the natural sciences, social sciences and the humanities. Scientometrics, 68 (3), 519-533

Moed, H. F. (2006): Bibliometric ranking of World Universities CWTS Report 2006-01 Leiden.

Moed, H. F. (2005): Citation Analysis in Research Evaluation. Springer. Netherlands.

Moros, A., y Bordons, M. (2003): La memoria de actividad como fuente de información bibliométrica en el estudio de una Escuela Politécnica Superior. Revista Española de Documentación Científica, 26 (2): 143-161.

Narin, F.; Stevens, K., y Whitlow, E. S. (1991): Scientific cooperation in Europe and the citation of multinational co-authored papers. Scientometrics, 21 (3), 313-323.

Olmeda-Gómez, C.; Ovalle-Parandones, M. A.; Perianes-Rodríguez, A., y Moya-Anegón, F. (2008): Impacto internacional de la investigación y la colaboración científica de las universidades de Cataluña. Revista Española de Documentación Científica, 31 (4): 591-611.

Persson, O.; Glänzel, W., y Danell, R. (2004): Inflationary bibliometric values: the role of scientific collaboration and the need for relative indicators in evaluative studies. Scientometrics, 60 (3), 421-432.

SJTU (2005): Academic ranking of world universities 2007. Shanghai Jiao Tong University, Institute of Higher Education. Available at http://www.arwu.org/rank/2007/ ARWU2007_102-202.htm.

THES (2007): World University Rankings. 2007. http://www.thes.co.uk/worldrankings.

Thijs, B.; Glänzel, W. (2008): A structural analysis of publication profiles for the classification of European research institutes. Scientometrics, 74 (2): 223-236.

Torres-Salinas, D.; Delgado Lopez-Cozar, E., y Jiménez-Contreras, E. (2009): Análisis de la producción de la Universidad de Navarra en revistas de Ciencias Sociales y Humanidades empleando rankings de revistas españolas y la Web of Science. Revista Española de Documentación Científica, 32 (1): 22-39. 
Van Raan, A. F. J. (2005): Fatal Attraction: Conceptual and methodological problems in the ranking of universities by bibliometric methods. Scientometrics, 62 (1): 133-143.

Zitt, M.; Ramanana-Rahary, S.; Bassecoulard, E., y Lavilla, F. (2003): Potencial sciencetechnology spill-overs in regions: an insight on geographic co-location of knowledge activities in the EU. Scientometrics, 57 (2): 295-320. 


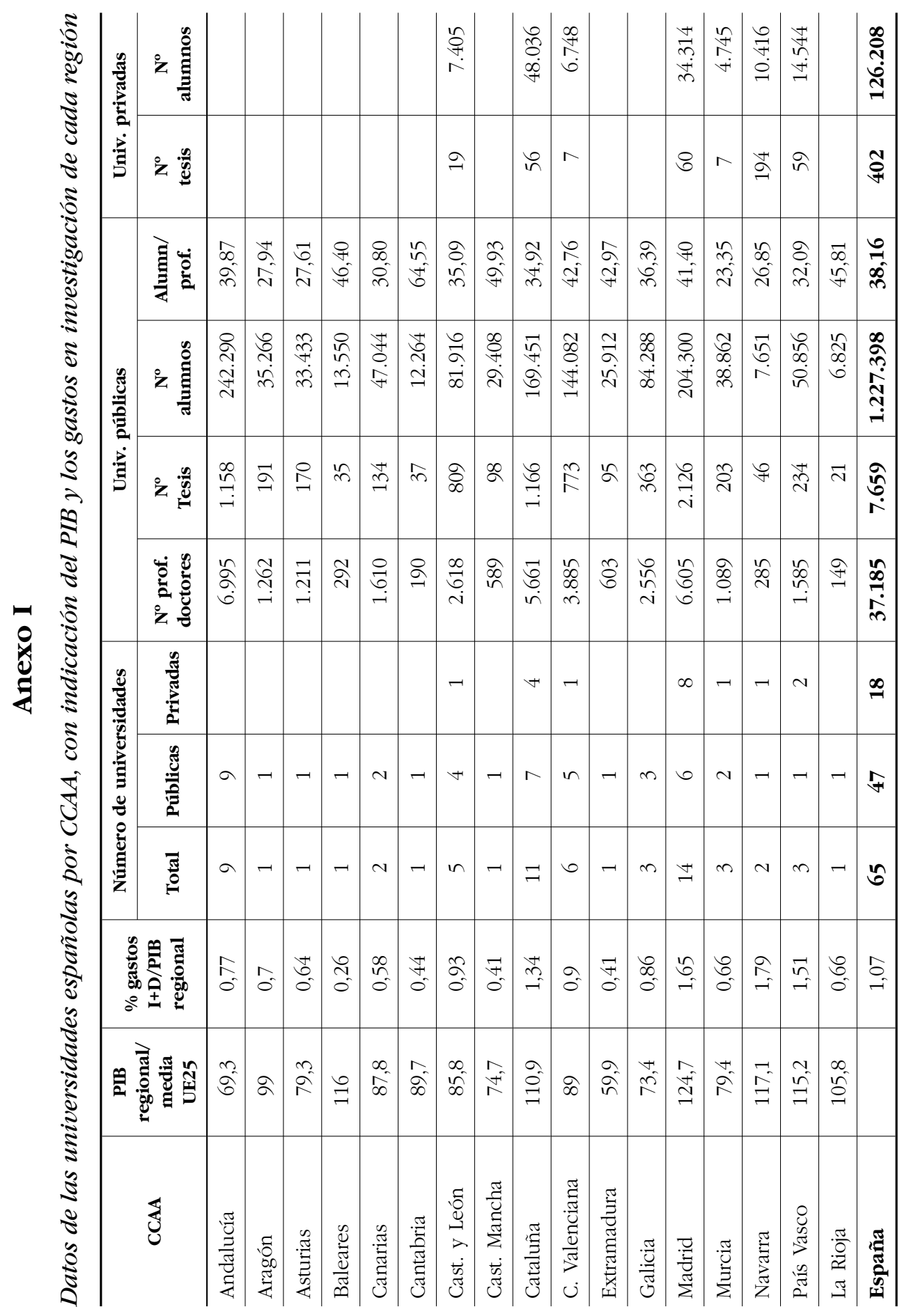




\section{Anexo II}

\section{Abreviaturas de las Universidades públicas españolas usadas en el estudio}

\begin{tabular}{|c|c|c|c|}
\hline Universidad & Abreviatura & Universidad & Abreviatura \\
\hline Univ. Autónoma de Barcelona & $\mathrm{UAB}$ & Univ. de Lérida & UDL \\
\hline Univ. Autónoma de Madrid & UAM & Univ. de Málaga & UMA \\
\hline Univ. Carlos III de Madrid & UC3 & Univ. de Murcia & UMUR \\
\hline Univ. Complutense de Madrid & $\mathrm{UCM}$ & Univ. de Oviedo & $\mathrm{OV}$ \\
\hline Univ. de Alcalá de Henares & $\mathrm{UAH}$ & Univ. de Salamanca & USAL \\
\hline Univ. de Alicante & UA & Univ. de Santiago de Compostela & USC \\
\hline Univ. de Almería & ALME & Univ. de Sevilla & US \\
\hline Univ. de Barcelona & UB & Univ. de Valencia & UVA \\
\hline Univ. de Burgos & UBU & Univ. de Valladolid & VALL \\
\hline Univ. de Cádiz & UCAZ & Univ. de Vigo & VIG \\
\hline Univ. de Cantabria & UCA & Univ. de Zaragoza & $\mathrm{UZ}$ \\
\hline Univ. de Castilla la Mancha & UCIM & Univ. del País Vasco & UPVEUH \\
\hline Univ. de Córdoba & UCOR & Univ. Jaume I & JAU \\
\hline Univ. de Coruña & UCN & Univ. Miguel Hernández & UMH \\
\hline Univ. de Extremadura & UEX & Univ. Pablo de Olavide & UPO \\
\hline Univ. de Gerona & GE & Univ. Politécnica de Cartagena & UCTG \\
\hline Univ. de Granada & UG & Univ. Politécnica de Cataluña & UPC \\
\hline Univ. de Huelva & UHU & Univ. Politécnica de Madrid & UPM \\
\hline Univ. de Jaén & JAEN & Univ. Politécnica de Valencia & UPV \\
\hline Univ. de la Laguna & LAG & Univ. Pompeu Fabra & UPF \\
\hline Univ. de la Rioja & URIOJ & Univ. Pública de Navarra & UPNV \\
\hline Univ. de las Islas Baleares & UIB & Univ. Rey Juan Carlos & URJC \\
\hline $\begin{array}{l}\text { Univ. de las Palmas de Gran } \\
\text { Canaria }\end{array}$ & UPGC & Univ. Rovira i Virgili & URIV \\
\hline Univ. de León & LEON & & \\
\hline
\end{tabular}

